\title{
Informatic Tools and Approaches in Postmarketing Pharmacovigilance Used by FDA
}

\author{
Joyce Weaver, ${ }^{1,2}$ Mary Willy, ${ }^{1}$ and Mark Avigan'
}

Received 24 September 2007; accepted 21 November 2007; published online 25 January 2008

\begin{abstract}
The safety profile of newly approved drugs and therapeutic biologics is less well developed by pre-marketing clinical testing than is the efficacy profile. The full safety profile of an approved product is established during years of clinical use. For nearly 40 years, the FDA has relied on the voluntary reporting of adverse events by healthcare practitioners and patients to help establish the safety of marketed products. Epidemiologic studies, including case series, secular trends, case-control and cohort studies, are used to supplement the investigation of a safety signal. Ideally, active surveillance systems would supplement the identification and exploration of safety signals. The FDA has implemented a number of initiatives to help identify safety problems with drugs and continues to evaluate their efforts.
\end{abstract}

KEY WORD: postmarketing product surveillance.

\section{POSTMARKETING PRODUCT SURVEILLANCE}

The Food and Drug Administration (FDA) is responsible for making sure marketed drugs and biologics are both efficacious and safe. At the time of FDA approval of drugs and therapeutic biologics, there is an imbalance in what is known about the efficacy and safety of the products. The efficacy data are robust; efficacy is well established by the randomized clinical trial data submitted to support the new drug application (NDA) or biologic licensing application (BLA). Less is known about the safety of newly approved drugs because clinical trials are not powered to detect rare drug-induced adverse events. Little is known about the safety of the products in a diverse (in age, ethnicity, gender, and comorbidities) population because the clinical trial subjects often do not reflect the more diverse patient population in which the products will be used after approval. Often narrow indications are studied in clinical trials, but the reasons for use broaden when the product is introduced into clinical practice. The clinical studies are short in duration, and do not reflect chronic use that may occur in clinical practice. The full safety profile of the drug emerges over a period of years of use in clinical practice as the drug is used in a wider and more diverse patient population than occurred in pre-marketing clinical trials. The full safety profile of an approved product is established during years of clinical use as healthcare practitioners and patients

\footnotetext{
${ }^{1}$ Office of Surveillance and Epidemiology, Center for Drug Evaluation and Research, Food and Drug Administration, 10903 New Hampshire Ave., Silver Spring, MD 20903-0002, USA.

${ }^{2}$ To whom correspondence should be addressed. (e-mail: joyce. weaver@fda.hhs.gov)
}

report their adverse experiences with the product and as the product is studied in epidemiologic studies.

\section{FDA'S ADVERSE EVENT REPORTING SYSTEM (AERS)}

The reports of adverse events that occur in clinical practice are voluntarily reported by healthcare practitioners and patients to the pharmaceutical manufacturers or directly to the FDA. These case reports are placed into a computerized safety database at the FDA, the Adverse Event Reporting System (AERS), a database that contains spontaneously reported cases. AERS, a computerized database which began nearly 40 years ago, is a database containing over 3 million case reports. With the spontaneously reported cases that are stored within the AERS database, the FDA gathers safety data relatively inexpensively to allow the detection of events not seen in clinical trials (1). Exploration of the cases stored within AERS is especially useful for detecting rare events associated with a drug product (2). Because a healthcare practitioner is more likely to suspect that an event is attributable to drug exposure if the event occurs fairly quickly after drug exposure, events with short latency following drug exposure are more likely to be reported to the FDA and therefore stored within AERS.

Because AERS comprises spontaneously reported case reports, it has important limitations as a tool in detecting and exploring drug safety problems. The database contains duplicate reports because more than one person may report the same event. Although duplicate reports may be submitted for some events, a bigger problem with AERS is extensive underreporting, the true extent of which is not known (1). Because busy healthcare practitioners are submitting the bulk of the reports 
contained within AERS, the quality of the reports is variable; the reports often lack data critical for the evaluation of the event.

The cases submitted to AERS may be viewed as reflecting adverse effects relating to real-world use of the products in clinical practice. The patient population may be more inclusive than the patient population the product was tested on in clinical trials. The patients may include a more diverse patient population in terms of age, gender, ethnicity, and medical histories. The product may be used for wider indications than studied, and the use of medication may be chronic. Because the patients who use the product may have complex medical histories, the AERS cases tend to be complicated. The complicated nature of the postmarketing cases poses significant challenges to FDA scientists in attributing the adverse events to exposure to a particular drug product.

The actual number of adverse events occurring in people who receive a drug product and the number of patients who have been exposed to a drug product in the population are not known, and so incidence rates cannot be calculated from AERS data for drug-induced adverse events. Furthermore, it can be difficult to attribute causality for events to drug exposure if the adverse event occurs frequently in the population in the absence of drug exposure. For example, myocardial infarction may be difficult to attribute to drug exposure using AERS data alone, because myocardial infarction is a common event in the general population and therefore may not be reported.

\section{USING AERS TO DISCOVER AND EXPLORE SAFETY ISSUES}

Safety evaluators, most of whom are clinical pharmacists, perform the frontline postmarketing safety surveillance for approved drugs and therapeutic biologic products. The main mission of the safety evaluators is to identify and monitor safety signals for the products. The adverse event case reports are entered into the AERS database, and each report of an unexpected event (that is, an event not described in the product labeling) with a serious outcome (for example, death, hospitalization, or disability) is triaged to a safety evaluator. Select other reports are sent to the safety evaluators as well, including some reports that are sent on an expedited basis to the FDA as a result of increased surveillance on an issue of special concern for the product. The reports are sent to a virtual inbox for each safety evaluator. In 2006, more than 218,000 such reports were forwarded to the FDA safety evaluators (3). The safety evaluators monitor the AERS inbox on a daily basis reading the serious, unexpected reports that have been submitted for the drug products.

Although the safety evaluator's AERS inbox is a primary source of postmarketing safety data, the safety evaluators receive postmarketing safety data from other sources as well. For each drug product, the safety evaluators receive mandatory periodic safety reports prepared by the product's manufacturer. The periodic safety reports summarize all the case reports submitted to the manufacturer for a product. Safety issues can be raised other ways as well; for example, the published medical literature or safety data in a New Drug Application might raise a safety question. A safety question can be raised through an inquiry to the FDA from a clinician, patient, or a member of Congress.

Data mining can help in detecting safety signals. The FDA uses data mining to screen the AERS database using a Bayesian protocol for the presence of disproportionately large adverse event-drug product pairs; that is, the database is screened to detect the presence of an unexpectedly high number of reports of a given adverse event for a drug product. The FDA uses the empirical Bayes multi-item gamma Poisson shrinker (MGPS) data mining protocol. MGPS incorporates numerous stratification steps to minimize false positive signals. Details on the MGPS algorithm used by the FDA have been published elsewhere (4).

The presence of an unexpectedly high number of reports for a given event for a drug product does not mean necessarily that the drug product causes the event, nor does the absence of a data mining safety signal mean there is not a safety problem. The data must be evaluated to determine whether or not the disproportionately high adverse eventdrug product pair represents a true safety signal for the drug product (5). The biases present in the AERS database are still present when the database is "mined" for disproportionately high adverse event-drug product pairs, and these biases must be considered when evaluating the data. Data mining information from screening the AERS database generate hypotheses that are examined to determine the validity of the potential safety signal and to determine the need for additional analyses.

After a safety question is raised, the safety evaluator has a number of options to pursue the safety issue. These options include screening and analyzing AERS cases, requesting and analyzing additional clinical details from patients and healthcare practitioners who reported cases of interest, analyzing data mining information, and evaluating similar cases reported in the medical literature. Often, the first step in pursuing a safety issue is searching AERS for case reports that may speak to the safety issue. Safety evaluators search the AERS database using the medical terminology MedDRA, the Medical Dictionary for Regulatory Activities. MedDRA is an international hierarchical medical terminology that is used to code events within AERS. The safety evaluator searches AERS using the MedDRA hierarchy of terms (i.e., preferred terms, higher level terms, high level group terms, or system organ class terms) to broaden or narrow the search (6).

When safety evaluators gather cases from AERS, they usually find a range of cases in terms of information quality, including cases with a very complete description and documentation, and cases with very little supporting clinical information. Ideally, at least some case reports contain complete information including a complete description of the event, what product the reporter thinks caused the event, other products the patient was taking, patient characteristics, medical conditions, risk factors, documentation of the diagnosis, information on whether the event abated when the product was discontinued (dechallenge), and information on whether the event recurred if the product was reintroduced (rechallenge).

The safety evaluator examines the case reports to determine if there are other factors that may explain the event, or if, indeed, the drug product is the likely cause. For any individual case report, it is rarely possible to know 
whether the event was caused by the drug product. Nevertheless, the safety evaluator assesses the strength of the evidence for causality, looking for the temporal relationship between the use of the drug and the event, drug-disease interactions, other drugs the patient was taking that may have contributed to the event, and supportive clinical and laboratory findings. Additionally, the safety evaluator examines cases for dechallenge and rechallenge information, the biologic plausibility of the event, and known drug class effects. The safety evaluator may examine pre-marketing data to see if the event was observed in clinical testing.

Safety evaluators examine the case reports in AERS for commonalities in an adverse event reported for a drug product. The safety evaluator looks at the cases together for the ages of the patients, the sex of the patients, the time to onset of the adverse event, whether there is a dose-response relationship, and whether there are risk factors that may make patients more likely to experience the event.

How well does a spontaneous reporting system like AERS handle safety issues? For rare, easily diagnosed events with short latency following drug exposure, AERS is an efficient database to examine drug-induced adverse events (2). For events confounded by the indication for use, for events that occur frequently in patients not exposed to the drug product, and for events that are not easily diagnosed, AERS is not an efficient database to examine the druginduced adverse events. The impact of these factors on the capability of AERS to discover and explore safety signals can be illustrated by examining several drug-related safety issues that the FDA has confronted.

Terfenadine (Seldane) was approved in 1985 for the relief of symptoms associated with seasonal allergic rhinitis and finally was withdrawn from the market in 1998 because of its role in causing cardiac arrhythmias, notably QT prolongation and torsade de pointes. The reporting of QT prolongation to the FDA for terfenadine followed an atypical pattern. The FDA usually receives the most adverse event reports for a drug within the first few years of marketing (7). The reports for QT prolongation with terfenadine did not follow this pattern, with the most reports of QT prolongation received in 1992, seven years after the drug's approval. It appears that there was a delay on the part of attending physicians in the recognition of the role of terfenadine in producing arrhythmias. The first step in reporting a safety issue is diagnosis of the medical event. This was not accomplished easily in the case of terfenadine-induced arrhythmias because patients taking terfenadine generally were not being monitored with EKGs. Even when a rhythm abnormality became clinically apparent, the diagnosis of the specific arrhythmia was still difficult. In fact, AERS contains reports of syncope for terfenadine that probably were cases of torsade de pointes. Finally, after the arrhythmia is diagnosed, to be reported to the FDA as a drug-related adverse event, the physician must have a suspicion that the event was secondary to exposure to a drug. In this case, the diagnosis of the arrhythmia and attribution to terfenadine were problematic. Because of these factors, this signal was slow to appear in AERS data.

Salmeterol (Serevent) was approved in 1994 for the maintenance treatment of asthma. Early in the marketing of salmeterol the FDA received postmarketing reports of asthma exacerbation with use of the product. It is expected that some asthma patients will experience exacerbation of asthma, even while on therapy. The cases reported for salmeterol were confounded by the indication for the use of the product; that is, the patient received the drug for asthma, and so it is difficult to determine whether the asthma exacerbation was caused by salmeterol, or was an expected manifestation of the patient's underlying disease. The question to be answered in evaluating the reports is whether there is something being experienced by the patients using salmeterol that is out of line with what is expected, not only with the drug, but with the underlying disease. The essential question is whether there is a differential rate of exacerbations of asthma that is attributable to the drug. By the nature of the uncontrolled data within AERS, the AERS database cannot answer this question; AERS cannot establish the comparative frequency of an adverse event. Because AERS could not answer the safety question, a large safety study was undertaken to explore the safety issue. Although the study did not answer the safety question fully, the study was stopped when an interim analysis showed significant increases in respiratory-related and asthma-related deaths and combined asthma-related deaths or life-threatening experiences in the group receiving salmeterol (8).

Valdecoxib (Bextra) is a COX-2 selective NSAID that was approved in 2001 and was first marketed in early 2002. Early in marketing the FDA received a number of cases of Stevens-Johnson syndrome and toxic epidermal necrolysis temporally related to the use of this product. This information was quickly incorporated into the labeling for the product.

What were the characteristics of this issue that lent it to be explored via AERS? This is the type of issue that AERS handles very well. First, the serious skin events are rare, easily diagnosed events, with short latency from the time the drug was started; secondly, because Stevens-Johnson syndrome is well recognized as a drug-induced event, the practitioners attributed the reactions to valdecoxib; thirdly, the practitioners reported the events to the manufacturer and to the FDA. Finally, although FDA scientists seldom make comparisons of reporting rates between products, because of the similarity between the drugs in indication for use, time on the market, and year of introduction to the market, it was reasonable to compare the reporting rates for this event for valdecoxib and other drugs in the class at a similar point in marketing. This comparison showed that the reporting rate for Stevens-Johnson syndrome/toxic epidermal necrolysis for valdecoxib was 8-9 times that of celecoxib and approximately 25 times that of rofecoxib and the background rate (9). Based on these data, FDA scientists concluded that these events occurred more frequently with valdecoxib compared to other products in the drug class.

A second safety issue with valdecoxib is an example of an issue that is not a good issue to study in AERS. Valdecoxib is associated with thromboembolic events (TEE), fairly common events even in patients not exposed to valdecoxib. Because of the high background rate of TEE, it is difficult to explore this using AERS data.

For both the salmeterol asthma case study in which the cases are confounded by indication and the TEE valdecoxib case study where there is a high background rate of the event in the general population, AERS is of limited use in exploring the safety issue. 


\section{EPIDEMIOLOGIC STUDIES}

Passive surveillance is helpful in identifying a possible adverse event and drug association, but as described above, there are a number of limitations. Underreporting is known to be a common problem with passive surveillance (7). Reporting also varies over time and may be affected by secular trends (7). There can be differences in the diagnostic terminology used. And finally, the analysis of passive surveillance data can not yield an incidence rate.

Rates obtained from passive surveillance are often referred to as reporting rates and usually reflect the number of cases reported divided by the number of patient exposures. As discussed the numerator will be an underestimate of the true number of cases, although media attention can lead to an increase in reported cases. The estimated denominator is also difficult to obtain, since the number of patients truly exposed and for how long is not easily determined.

Often reporting rates will be compared to background rates and possibly to the reporting rates with similar drugs to determine if there is a safety signal. Background rates in the population or in a special population (like pediatrics) might be used for the comparison, but are often difficult to obtain. Comparisons to other drugs must be done with care since the reporting for each drug is influenced by the length of time on the market, the level of manufacturer diligence to identify cases, and whether one drug has had differential media attention related to the event of interest. Factors that are considered when analyzing reporting rates are the magnitude of the differences, whether the findings are consistent when looking at less severe forms of the event, and whether information from the clinical trial studies and literature reports support the findings.

As part of the investigation of a safety signal, there are times when epidemiologic studies can provide useful information. The types of studies that might be used postmarketing include case series, secular trends, case-control and cohort studies (10-12). Case series are generally a description of a group of patients experiencing similar adverse events or drug exposure with no control group for comparison. The study of secular trends in certain outcomes can be linked to the use of certain drugs in some defined population to help with the initial exploration of a hypothesis. Since the secular trend data are not linked to individual patients, positive findings must be confirmed with more systematic studies. Case-control studies compare identified cases with a defined outcome to a group of controls without the outcome; exposure to drugs can be compared between each group. Case-control studies are useful for rare diseases and when there is interest in multiple causes for a given outcome. Cohort studies study defined populations over time to look for outcomes of interest. Cohort studies generally compare people exposed to a drug to those who are not exposed. Cohort studies benefit from reduced recall bias (accuracy of the recalled information) since information is generally collected prospectively. But cohort studies usually require large numbers of people and can take an extended period to complete.

Many epidemiologic studies require large numbers of patients to provide sufficient statistical "power" for the analysis to be worthwhile $(13,14)$. The populations used for pharmacoepidemiologic studies should ideally be able to link medical, pharmacy and demographic information to allow a complete analysis. A number of different resources are available that link relevant information, some of the information being available electronically (through insurance claims) and other information requiring some manual review of medical records (such as details about the sequence of events, other risk factors). These databases have the advantage of providing detailed information on patients for long periods of time, to allow for retrospective cohort and casecontrol studies. There are always concerns, though, about the generalizability of these data to other populations.

\section{ACTIVE SURVEILLANCE AND OTHER ENHANCED REPORTING SYSTEMS}

Active surveillance is a pharmacoepidemiologic tool that has received attention as a drug safety tool. Active surveillance can be defined as the regular periodic collection of case reports from health care data systems. The process of active surveillance may be used to identify a new drug safety signal or to validate drug safety signals identified through passive surveillance. Active surveillance can be structured in a number of different ways. When a new drug is marketed, an active surveillance program might be established to monitor prospectively for safety signals that were too rare to appear in the typical clinical trial or, in some cases, to look for events that were hinted at in the clinical trials, but that did not have sufficient statistical power to definitively identify; this type of surveillance is drug-based. Certain settings like emergency departments (ED) can be used to look to see what drugrelated events are presenting to ED (15). Anaphylaxis is a good example of a critical event that would be seen in the ED and might be related to a drug. Lastly, monitoring certain diseases (or outcomes) might be initiated to determine if there are any drug related events. For example, registries for liver failure or aplastic anemia might collect information on drug exposure to determine if there are unusual numbers of a certain event in patients receiving a certain drug.

Although many would like to see the development of active surveillance systems there are several challenges that make their development difficult. First, an effective program requires the receipt of timely information that has been validated and comes from both in- and out- patient data sources. Additionally, an effective system should represent a large population. As healthcare systems become more automated these components should become more readily available making an effective system possible. Most would agree, though, that the perfect system is not currently in place.

Although there is no perfect example of a current active surveillance system, there are examples of programs that have components of an active surveillance system: National Electronic Injury Surveillance System-All Injury Program (NEISS-AIP), Natalizumab and Progressive Multifocal Leukoencephalopathy (PML), and NIH sponsored Drug Induced Liver Injury Network (DILIN). These systems make efforts to collect information prospectively as an active surveillance system might do, but for the most part lack efficient procedures to provide timely data and might better be referred to as enhanced surveillance systems. 


\section{ENHANCED SURVEILLANCE BASED ON CERTAIN SETTINGS}

\section{National Electronic Injury Surveillance System-All Injury Program (NEISS-AIP)}

The US Consumer Product Safety Commission (CPSC), the Centers for Disease Control and Prevention (CDC) and the Food and Drug Administration (FDA) have funded a nationally representative study of medication-related adverse events presenting to Emergency Department. The program collects data from a sample of EDs. Trained abstractors review ED medical records to identify patients diagnosed with a condition that is linked to a drug. This data source can be used to identify new safety signals or can also provide information about the public health impact of know adverse events. A recent summary of this program's active surveillance findings for 2004 and 2005 showed that allergic reactions were the most common adverse event treated in EDs followed by unintentional overdoses (15).

\section{ENHANCED SURVEILLANCE BASED ON TREATMENT WITH A SPECIFIC DRUG(S) AND INDICATION}

\section{Natalizumab and Progressive Multifocal Leukoencephalopathy (PML)}

In November 2004 natalizumab (Tysabri) was approved by FDA for the treatment of relapsing forms of multiple sclerosis (MS). Identification of an association between natazulimab with PML emanated from two postmarketing clinical studies to determine the safety and efficacy of this agent in patients with relapsing-remitting forms of the disease that were being performed by the manufacturer at the time of approval. In February 2005 the FDA received a report from the manufacturer of Tysabri of one confirmed fatal case and another suspected case of PML (later confirmed) in enrolled patients who had received Tysabri, each for over two years, in addition to treatment with IFN $\beta-1$ a. This led to the immediate suspension of marketing at the end of February, 2005 to permit an in-depth evaluation of the risk and benefit of this product for the treatment of relapsing MS. Subsequently, evaluation of another patient who died in a trial for the treatment of Crohn's disease confirmed a third case of PML. With a known denominator of approximately 3,000 patients who received the drug during clinical trials to treat MS or Crohn's disease it was possible to estimate a rate of PML in patients who received natazilumab of approximately $1 / 1,000(16,17)$. Because of the small number of PML cases it was not possible to definitively determine the relative contribution to risk by concomitant treatment with IFN $\beta$-1a or other immunosuppressive drugs.

The discovery of an initial PML signal in ongoing postmarketing clinical protocols of inception cohorts who have initiated treatment in pre-approval phase III studies was efficient and promptly reported to FDA. It highlights the utility that such a surveillance strategy has in the early detection of a safety signal, especially when treatment related risk for an AE may be dependent on time of treatment, total drug exposure or patient related susceptibility factors which may change over time. Inception cohort studies which track a pre-approval study population chronically exposed to a drug after approval can play a crucial role in the identification of risk that appears after a latent period of drug exposure. In addition, the opportunity to study risk is enhanced if treatment is given by specialized health care providers located in medical centers with a capacity to perform comprehensive patient follow-up, clinical assessment and meticulous record keeping.

In June 2006 FDA approved an application for resumed marketing of natazilumab with a special restricted distribution program linked to a Risk Management Plan called the TOUCH Prescribing Program which has been designed to minimized the risk, death and disability due to natalizumabassociated PML and promote informed risk benefit decisions for MS patients regarding this agent (18). Among the elements of this program is registration of all patients, prescribers, natazulimab infusion centers and their associated pharmacies. In addition, a plan has been developed to monitor, evaluate and measure risk factors associated with cases PML and other serious opportunistic infections and regularly report these to FDA. The TOUCH Prescribing Program is designed to provide a highly effective registry for PML which is based on drug exposure and relies on a highly specialized health care environment.

\section{ENHANCED SURVEILLANCE BASED ON SPECIFIC TOXICITIES/CLINICAL OUTCOMES}

\section{NIH Sponsored Drug Induced Liver Injury Network (DILIN)}

Although drug-induced liver injury (DILI) is a rare clinical event, it is the most common cause of acute liver failure in the US. Idiosyncratic forms of DILI, which are not predictable, have been linked to only a subset of therapeutic products, and occur only in susceptible individuals. Both mechanisms of liver injury and factors of individual susceptibility are poorly understood. Unfortunately, in some instances DILI has presented a barrier in drug development. Moreover, it has been a leading cause of medication withdrawal or lack of approval by FDA. To characterize cases and develop a better understanding of the epidemiology and pathogenesis of this problem a number of studies or registries have been initiated in the US and Europe. The NIH has funded a consortium of 5 US medical centers to perform retrospective and prospective studies of patients identified at these centers with this condition (19). The DILIN prospective study seeks patients with suspected DILI not caused by acetaminophen that has occurred within 6 months of enrollment. Referred patients with clinical case material are extensively evaluated and thorough differential diagnoses and expert causality assessments are performed. Biological specimens for each patient including serum, urine, lymphocytes and DNA are routinely collected for future studies. Since 2004, over 200 patients have been enrolled in the prospective study (20). So far, the leading drug class associated with identified and referred cases of idiosyncratic DILI are antimicrobials. Although identification and inferences of risk associated with drugs identified by this study may be subject to a number of biases, DILIN has a potential to develop into a useful disease based active 
surveillance system for an important category of drug related adverse events in the U.S.

\section{OTHER SAFETY EFFORTS}

\section{Pooled Analysis or Meta-analysis of Randomized Clinical Trial Safety Datasets}

Causal attribution to drug exposure for adverse events with high background rates in the same patient population is often difficult to determine. One approach to addressing this problem is the analysis of randomized clinical datasets. After FDA approval of a new molecular entity based on review of pre-marketing studies, manufacturers often continue to perform randomized clinical trials, comparing the agent with placebo or active comparators. Often this is done in order to study other indications of use, drug effects in different patient populations, new dosaging protocols, etc. Because individual clinical trials are usually not sufficiently powered to detect and measure risk surrounding safety events which are either rare or superimposed on high background rates in the treated populations, a portfolio of randomized studies performed before or after initial marketing can be an invaluable resource to identify and assess these types of drug-related events. Pooled or trial based meta-analyses of the randomized clinical trial safety datasets to measure imbalances of these events may be especially useful. Although there are important limitations to consider in the analysis and interpretation of such measurements, a recent example illustrates the value of this approach for safety signal detection.

In the case of antidepressants (including SSRIs), review of integrated clinical trial (ICT) data from short-term studies in children and adolescents with major depressive disorder and other psychiatric disorders uncovered an increased risk of suicidal thinking and behavior (suicidality). Pooled analyses of 4 to 16 week placebo-controlled trials of 9 drugs (24 trials involving more than 4,400 patients) showed an increased risk for suicidal thinking or behavior in these age groups during the first few months of treatment (21). The average risk of such events in pediatric patients receiving antidepressants was $4 \%$, twice the placebo associated risk of $2 \%$ (21). A similar analysis revealed that this risk extends to young adults ages 18 to 24 during initial treatment, generally during the first one or two months of treatment (22).

Important issues can surface in the interpretation of postmarketing randomized clinical studies. Establishing whether relative risk measures derived from a meta-analysis of clinical trials or individual studies reflects actual drugrelated risk can be challenging. In assessing study outcomes one must consider multiple factors including consistency of endpoints and measurements of other known risk factors for the event of interest. In addition, it may be important to determine whether active comparator groups are sufficiently characterized to draw comparative conclusions with regards to risk. Finally, the studies should be sufficiently powered and employ reliable methods of adverse event ascertainment, classification and reporting. In the presence of meta-analyses and analyses of clinical studies with disparate results, careful attention must be given to such parameters in order to draw conclusions about the relative contribution of each set of results to an overall assessment of a safety signal and/or quantitative measurement of risk. This will be especially important as more cumulatively acquired postmarketing safety data from randomized clinical trials are made available for scrutiny by academic experts and the FDA.

\section{Regular Safety Reviews of New Molecular Entities (NMEs)}

Beyond awareness of the datasets and analytic tools that form the foundations of effective pharmacovigilance, it is critical to reinforce standard review processes to achieve consistent and comprehensive safety evaluations of drugs. One recent initiative that is being developed by the Office of Surveillance and Epidemiology and the Office of New Drugs for this purpose is a pilot program to systematically evaluate the safety profiles of NMEs on a regularly scheduled basis (23). Postmarketing evaluations of NMEs will incorporate review of multiple streams of data including AERS, data mining analysis, epidemiologic datasets, postmarketing clinical trial information, and Periodic Safety Update Reports (PSURs) or U.S. Periodic Reports, to identify potential safety concerns early in the product life cycle which require in-depth evaluations.

\section{CONCLUSIONS}

The identification of safety signals after drugs have been approved for marketing is a complex process that requires extensive resources. Efforts continue at the FDA to improve the efficacy and effectiveness of its drug safety program so that safety problems are identified and in a timely fashion. Ongoing enhancements in postmarketing studies and increasing access to multiple streams of data pose many new challenges in the timely and accurate detection and assessment of drug safety.

\section{REFERENCES}

1. A. P. Fletcher. Spontaneous adverse drug reaction reporting vs event monitoring: a comparison. J. R. Soc. Med. 84(6):341-344 (1991).

2. V. Pinkston, and E. J. Swain. Management of adverse drug reaction and adverse event data through collection storage and retrieval. In M. D. B Stephens, and P. A. Routledge (eds.), Detection of New Adverse Drug Reactions: Macmillan References LTD., London, UK, 1999, pp. 282.

3. FDA Office of Surveillance and Epidemiology, unpublished data, 2006.

4. A. Szarfman, S. G. Machado, and R. T. O’Neill. Use of screening algorithms and computer systems to efficiently signal higherthan-expected combinations of drugs and events in the US FDA's spontaneous reports database. Drug. Saf. 25(6):381-392 (2002).

5. M. Hauben, and X. Zhou. Quantitative methods in pharmacovigilance: focus on signal detection. Drug. Saf. 26(3):159-186 (2003).

6. MSSO website. URL http://www.meddramsso.com/MSSOWeb/ index.htm.

7. S. R. Ahmad. Spontaneous reporting in the United States. In B. L. Strom (ed.), Pharmacoepidemiology: Wiley and Sons, West Sussex, England, 2005, pp. 136-159.

8. H. S. Nelson, S. T. Weiss, E. R. Bleecker, S. W. Yancey, and P. M. Dorinsky. SMART Study Group. The Salmeterol Multicenter Asthma Research Trial: a comparison of usual pharmacotherapy for asthma or usual pharmacotherapy plus salmeterol. Chest. 129 (1):15-26 (2006).

9. L. La Grenade, L. Lee, J. Weaver, R. Bonnel, C. Karwoski, L. Governale, and A. Brinker. Comparison of reporting of 
Stevens-Johnson syndrome and toxic epidermal necrolysis in association with selective COX-2 inhibitors. Drug. Saf. 28 (10):917-924 (2005).

10. B. L. Strom. Study designs available for pharmacopeidemiology studies. In B. L. Strom (ed.), Pharmacoepidemiology: Wiley and Sons, West Sussex, England, 2005, pp. 17-28.

11. L. Gordis. Epidemiology, W.B. Saunders Company, Philadelphia, 1996.

12. K. J. RothmanS. Greenland. Modern epidemiology, 2nd ed, Lippincott-Raven, Philadelphia, PA, 1998.

13. B. L. Strom. Study overview of automated databases in pharmacoepidemiology. In B. L. Strom (ed.), Pharmacoepidemiology: Wiley and Sons, West Sussex, England, 2005, pp. 219-222.

14. S. Hennessy. Use of health care databases in pharmacoepidemiology. Basic Clin. Pharmacol. Toxicol. 98:311-313 (2006).

15. D. S. Budnitz, D. A. Pollcok, K. N. Weidenbach, A. B. Mendelsohn, T. J. Schroeder, and J. L. Annest. National surveillance of emergency department visits for outpatient adverse drug events. JAMA 296:1858-1866 (2006).

16. Natalizumab Advisory Committee, March 2006: http://www.fda. gov/ohrms/dockets/ac/06/slides/2006-4208S1-Slide-Index.htm, last accessed July 9, 2007.
17. T. A. Yousry, M. Habil, E. O. Major, C. Ryschkewitsch, G Fajhle, S. Fiscer, J. Hou, B. Curfman, K. Miszkiel, N. MuellerLenke, E. Sanches, F. Barkhof, E. Radue, H. R. Jager, and D. B. Clifford. Evaluation of patients treated with Natalizumab for progressive multifocal leukoencephalopathy. N. Engl. J. Med. 354:924-933 (2006).

18. Natalizumab information: http://www.fda.gov/cder/drug/infopage/ natalizumab/default.htm, last accessed August 10, 2007

19. J. H. Hoofnagle. Drug-induced liver injury network (DILIN). Hepatology 40:773, 2004 (2004).

20. N. Chalasani, R. Fontana, P. B. Watkins, H. L. Bonkovsky, T. Davern, J. Serrano, and J. Rochan. Drug-induced liver injury network (DILIN) prospective study initial results. Am. J. Gastroenterol. 101:S168, 2006 (2006).

21. T. A. Hammad, T. Laughren, and J. Racoosin. Suicidality in pediatric patients treated with antidepressant drugs. Arch. Gen Psychiatry 63:332-339 (2006).

22. Anti-depressant December 2006 advisory committee http://www. fda.gov/ohrms/dockets/ac/06/slides/2006-4272s1-00-index.htm; last accessed July 9, 2007.

23. Future of Drug Safety; FDA's Specific Safety Initiatives; upgrading methods of benefit and risk analysis and risk management: www.fda. gov/oc/reports/iom013007.html, page 37, last accessed July 9, 2009. 